\title{
Hospital magnético: Escenario ideal que garantiza calidad del cuidado y la satisfacción laboral en enfermería.
}

\section{Hospital Magnet: ideal scenario to guarantee quality of care and job satisfaction of nurses}

Lic. Alicia Hernández Cantoral •

Mtra. Rosa A. Zárate Grajales.•

\section{Resumen}

Las instituciones del más alto nivel de calidad de los servicios de salud se denominan magnéticas por que han obtenido la acreditación desde esta perspectiva, logrando el reconocimiento social y fomentando la satisfacción del personal de enfermería. La Asociación Americana de Enfermeras (ANA) establece los tres criterios para que las instituciones sean certificadas como magnéticas: Ser un hospital con bajo índice de rotación hacia otras instituciones, tener una plantilla completa, y ser un hospital posicionado en un mercado laboral competitivo. Las características que se examinan en estos centros son la satisfacción personal y profesional, el estatus, el rol de la enfermera en la calidad del cuidado del paciente, la relación enfermera(o) paciente, reclutamiento y mantenimiento de las y los profesionales, la relación medico(a) enfermera(o), la descentralización de la toma de decisiones, el liderazgo clínico, autonomía y responsabilidad en el cuidado del paciente y flexibilidad de los horarios de trabajo. La investigación en organizaciones magnéticas ha demostrado baja tasa de morbi-mortalidad, bajo índice de estancia hospitalaria, aumento de la satisfacción de pacientes, aumento de la satisfacción del personal de enfermería, menor incidencia de accidentes de trabajo y gran atracción de enfermeras. No cabe duda que el reconocimiento de los "Hospitales Magnéticos" es la mejor iniciativa basada en la evidencia para mejorar el entorno de la práctica de enfermería, posicionando a las instituciones de salud como organizaciones excelentes.

- Maestranda del Programa de Maestría en Enfermería

-•Profesora del Programa de Maestría en Enfermería de la UNAM Asociado "C" ENEO

correspondencia: Alicia.golondrina@hotmail.com

RECIBIDO 17 DE NOVIEMBRE 2010

ENVIADO 3 DE DICIEMBRE 2010

ACEPTADO 13 DE ENERO 2011 


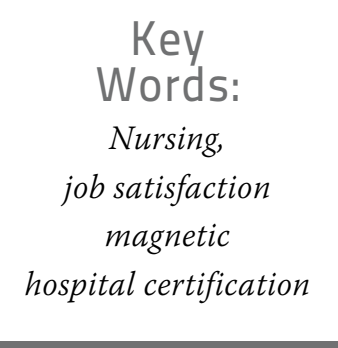

AbSTRACT

The institutions of the highest quality of health services are called magnetic because they have received accreditation from this perspective, achieving social recognition and fostering nurse satisfaction. The American Association Nursing (AAN) establishing the criteria for institutions to be certified as magnetic are three: Being a hospital with a low turnover rate to other institutions, have a fully staffed and positioned to be a hospital in a competitive job market. The features discussed in these centers are the personal and professional satisfaction, status, the role of the nurse in the quality of patient care, the nurse or patient recruitment and retention of the professional, the relationship medical-Nurse, decentralization of decision making, clinical leadership, autonomy and responsibility in patient care, ad flexible work schedules. Research has shown magnetic organizations: morbidity and mortality rate, low rate of hospital stay, increased patient satisfaction, increased satisfaction with the nursing staff, increased levels of nursing care, lower incidence of accidents and major attraction of nurses. No doubt that the recognition of "Hospitals Magnetic" is the best evidence-based initiative to improve the environment of nursing practice, positioning the health institutions as excellent organizations.

\section{INTRODUCCION}

Uno de los problemas que tienen los sistemas de salud a nivel mundial, y que preocupa no solo a los países desarrollados, es la escasez de enfermeras ${ }^{1}$, esta situación ha propiciado el análisis de centros hospitalarios, ocupados por atraer y retener a los profesionales de enfermería altamente capacitados y que buscan la satisfacción laboral adecuada, con base en estándares que permiten evaluar lo anterior como estrategia para asegurar la prestación de un servicio altamente calificado y la disminución de la alta rotación y el abandono de las enfermeras de los centros hospitalarios.

Existe evidencia suficiente en la literatura, que muestra que las instituciones del más alto nivel de calidad de los servicios de salud se denominan magnéticas por que han obtenido la certificación o acreditación desde esta perspectiva, logran el reconocimiento social y fomentan la satisfacción del profesional de enfermería.
A partir de esta denominación magnética, en EU comenzaron a surgir programas de acreditación para las instituciones sanitarias, como nivel de garantía de excelencia tanto en la satisfacción profesional como en la calidad de los cuidados.

Los criterios establecidos para que las instituciones fueran certificadas como magnéticas son tres: Ser un hospital con bajo índice de rotación hacia otras instituciones, tener una plantilla completa, y ser un hospital posicionado en un mercado laboral competitivo. ${ }^{2}$

Las características que se examinaron en estos hospitales fueron la satisfacción personal y profesional, el estatus, el rol de la enfermera en la calidad del cuidado del paciente, la relación enfermera(o) paciente, reclutamiento y mantenimiento de los y las enfermeras, la relación médica(o)-enfermera(o), la descentralización de la toma de decisiones, el liderazgo clínico, la autonomía y responsabilidad en el cuidado del paciente, y la flexibilidad de los horarios de trabajo, ${ }^{2}$ entre otras.

Actualmente existen estudios, donde se asocia la alta tasa de ausentismo y el sindrome de agotamiento profesional o burnout, entre el personal de enfermería, siendo el hospital el contexto en el que se genera. ${ }^{3}$ Esta tasa elevada de ausentismo apunta a ser una respuesta determinante de insatisfacción laboral.

Linda Aiken de la Universidad de Pennsylvania realizó un estudio en cinco países ${ }^{4}$ (Estados Unidos, Alemania, Canadá, Reino Unido y Nueva Zelanda) en 600 hospitales y con 15.000 enfermeras, aunque los países seleccionados corresponden a regiones diversas, considera que los problemas de enfermería son comunes. Dentro de lo más destacable, sustenta que Alemania tiene un porcentaje mucho más bajo de burnout; en Estados Unidos las enfermeras trabajan entre 36 y 40 hrs a la semana; y que de manera común, tienen en general 
doble empleo o jornada laboral. Estos datos arrojan que la profesión vive problemas derivados de la práctica y que esto está aunado a un grado de insatisfacción laboral. ${ }^{5}$

Es por eso que en América, las instituciones sanitarias han creado indicadores que permiten medir las variables que evalúan la satisfacción laboral de las enfermeras $y$ enfermeros de sitios acreditados como centros de excelencia, donde el personal de enfermería tiene autonomía destacable. ${ }^{6}$

En 1990 surge El Centro de acreditación de enfermeras americanas (ANCC) que creó el Programa de Reconocimiento de Hospitales Magnéticos (cuadro1) para reconocer la excelencia institucional ${ }^{7}$ basada en: La gestión, la filosofía y la práctica de los servicios de enfermería.

- La adherencia a los estándares nacionales para mejorar la calidad de los servicios de cuidados de los pacientes.

- El liderazgo de la enfermera gestora en dar soporte a la práctica profesional y competencia continua de las enfermeras.

- La comprensión y el respeto de la diversidad cultural y ética de los pacientes.

Esta perspectiva rescata la esencia del ser enfermera, puntualizando y orientando la práctica hacia una autonomía centrada en el cuidado. Por lo tanto los objetivos de los hospitales magnéticos comprenden:

- Identificar la excelencia de los cuidados de enfermería.

- Promover la calidad de las organizaciones y servicios en un entorno que apoye la práctica profesional de enfermería.

- Proveer el mecanismo para la divulgación de las mejores prácticas en los servicios de enfermería.

En este tipo de modelo existe una metodología que rescata la importancia del liderazgo, que tienen y deben de tener los profesionales de enfermería dentro de una institución, sin embargo existen algunos elementos esenciales en estas instancias, como son: la presencia de una política activa de formación y desarrollo profesional, una adecuada asignación de recursos humanos, la existencia de respeto y colaboración positiva con los equipos médicos, posibilidad de promoción y desarrollo profesional, competitividad destacable; en pocas palabras, elementos que permitan transformar las instituciones en centros idóneos para el trabajo profesional.

Las características que unifican y colocan en el punto de interés de las instituciones sanitarias con este enfoque, son 14 fuerzas, (cuadro 2) de los cuales ocho son los que actualmente se manejan como eje estándar del modelo.

Los investigadores en esta área han centrado 5 ejes $^{8}$ de los cuales, se derivan las fuerzas magnéticas (Figura 1), que a continuación se describen:

1. Liderazgo de transformación:

El líder debe ser creativo, visionario, inspirado, estratégico, comprometido, respetuoso, defensor, fiable, que trabaja hacia la estabilización de cada cambio de entorno, lo que requiere la habilidad de gestionar.

2. Empoderamiento estructural: Los líderes de transformación generan estructuras que soportan una práctica profesional fuerte donde la misión, visión y los valores de la organización vienen a la vida para controlar los resultados deseados de la población a la que atiende. En base a un plan estratégico, gestiona la información, recursos, soporte, políticas, sistemas y programas.

3. Práctica profesional ejemplar: modelo de práctica profesional, sistema de cuidados, proceso de gestión de recursos, cuidado interdisciplinar, responsabilidad, competencia y autonomía, ética, privacidad, seguridad y confidencialidad, diversidad, cultura de la seguridad, monitorización de la calidad y las mejoras.

4. Nuevo conocimiento, innovaciones y mejoras: $\mathrm{Se}$ centra en una práctica basada en evidencia, investigación, con mejora de la calidad (mejora de los procesos, análisis de beneficios, conformidad).

5. Resultados empíricos cualitativos: Por un marco en la estructura y procesos, buscando no “icómo lo haces?" sino "¿qué diferencia has creado?". Los resultados se tienen que catalogar en término de derivados clínicos, relacionados con enfermería, de personal, paciente, $y$ organizacionales. Se deberían establecer indicadores cuantitativos. Así como informes, reportes y notas (grupo central de indicadores enfermeros de calidad que incluyen estructura, proceso y resultados) que demuestren la organización magnética. 


\section{Enfermería Universitaria}

\section{Cuadro 1. Evolución del concepto "Hospitales Magnéticos".}

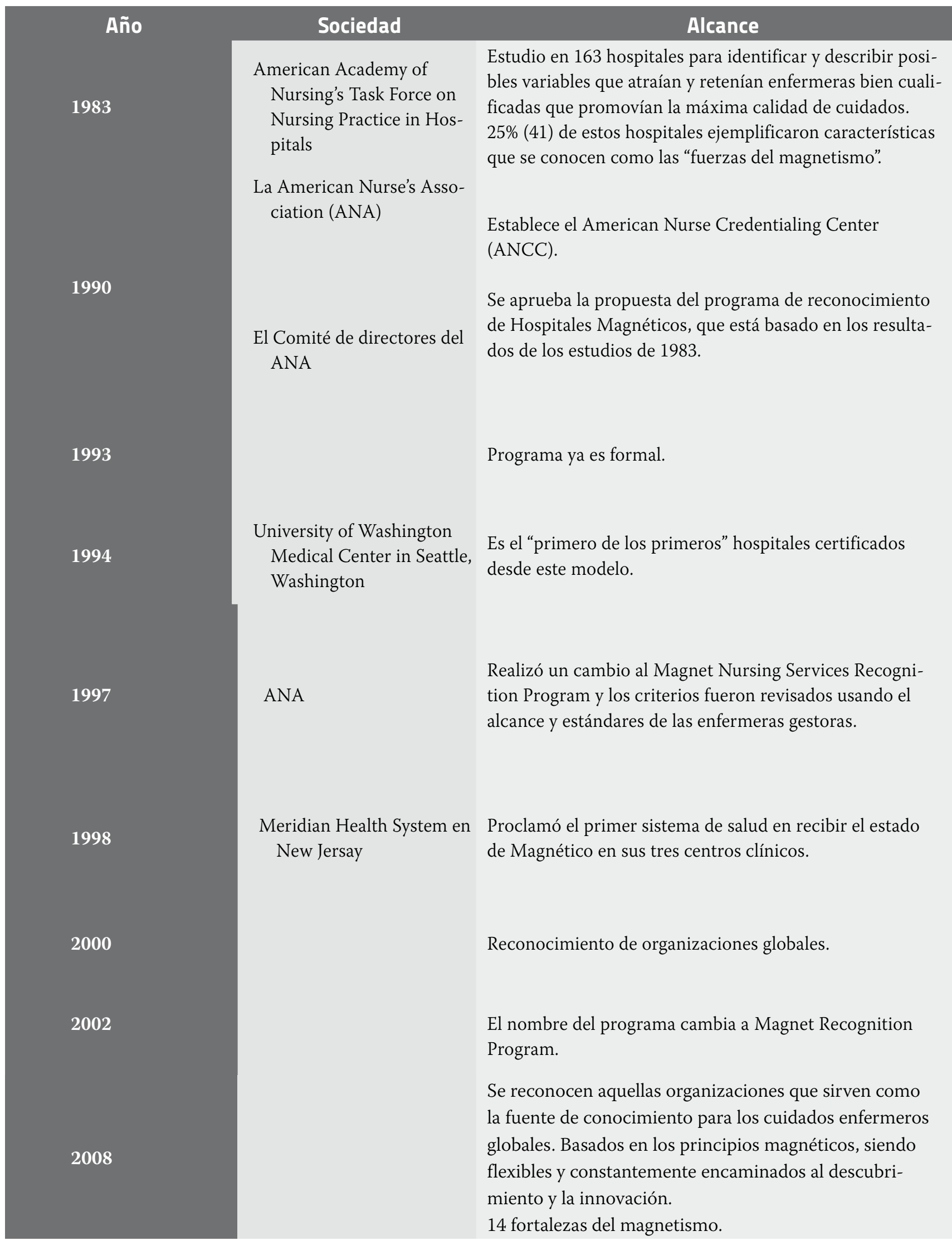


El valor que se le da a la certificación del Hospital Magnético está dirigido a crear una cultura en la organización de autonomía, respeto, metodología, trabajo en equipo, orgullo y mejores cuidados. Asegurando además la calidad y los resultados y establece un diferenciador de mercado.

Por otro lado la investigación en organizaciones magnéticas ha demostrado:

Una influencia muy fuerte de las direcciones de enfermería, menor tasa de morbi-mortalidad específicamente SIDA, bajo índice de estancia hospitalaria, aumento de la satisfacción de pacientes y del personal de enfermería, mejor percepción de la calidad de cuidados otorgados en el hospital, alta productividad, menor incidencia de accidentes de trabajo y gran atracción de enfermeras. ${ }^{9}$

Basado en el modelo de Donabedian ${ }^{10}$ la clasificación de necesidades de mejora de la calidad en estructura, proceso y resultado, el modelo de hospital magnético constituye una estrategia para mejora de la calidad. Los resultados se basan en la productividad, satisfacción, los logros y en las contribuciones. La estructura en la organización planificada, descripción de roles, recursos, equipo, sistemas, soporte y el proceso bajo parámetros de comunicación, desarrollo, reconocimiento, disciplina, educación y mejores relaciones.

Además con un departamento de enfermería adecuado y capacitado, se fomenta un mejor ambiente de trabajo, que son situaciones que han demostrado que mejoran la satisfacción laboral ofreciendo: Horarios de trabajo flexibles, buenas relaciones entre enfermeros(as) y médicos(as): trabajo en equipo, mejor gestión del hospital con participación de enfermería, no se actúa en contra de los propios criterios, se tiene un adecuado Programa de Inducción y "capacitación", y la aplicación de cuidados de enfermería basados en modelos innovadores. (Véase cuadro 2)

La clave del éxito en la automatización de los procesos clínicos y el Magnetismo complementan en forma paralela al proceso de desarrollo de una organización magnética, con un soporte centrado en un adecuado liderazgo ejecutivo, donde se estima que:

- El cambio no es una opción, es más bien, una expectativa

- Realizar un plan proactivo para anticipar la resistencia

- Liderazgo comprometido durante todo el ciclo

- No es un proyecto independiente, este debe estar liderado por un equipo multidisciplinar

- Se alinea con un plan estratégico y con imperativos corporativos

- Debe estar centrado por una gestión del cambio, una difusión e innovación de procesos

- Debe establecer objetivos de acuerdo con los gestores, y comunicar los beneficios de la realización

- La transparencia para alcanzar la calidad de atención

\section{Conclusiones}

Los atributos magnéticos constituyen una de las estrategias más sólidas para la mejora de los cuidados de salud y organización hospitalaria. Siendo una propuesta que se basa en la evidencia, para lograr reformas organizativas y definir políticas relativas a recursos humanos de enfermería en las instituciones de salud, es un planteamiento innovador que puede tener una aplicación como estrategia para potenciar tanto el entorno laboral como la calidad de los cuidados hacia los pacientes, ${ }^{12}$ Cuyos resultados son superiores a los de otros procesos de reingeniería hospitalaria.

Los Hospitales Magnéticos se caracterizan por tener una estructura horizontal, con poco personal supervisor, dando a los profesionales enfermeros mayor autonomía, control sobre la práctica y satisfacción laboral. En ellos se da una buena comunicación entre enfermeras y médicos, es decir, consideran a la enfermera como el eje fundamental del cuidado hospitalario, lo que garantiza la calidad de los cuidados y una mejora en los resultados de los pacientes.

El reconocimiento de los "Hospitales Magnéticos" es la mejor iniciativa basada en la evidencia para mejorar el entorno de la práctica de enfermería, en los resultados de una adecuada evolución de los pacientes y en la satisfacción tanto de usuarios como del personal institucional, posicionando a las instituciones de salud como organizaciones excelentes. ${ }^{8}$

Es un sistema sumamente promisorio para reducir los errores al brindar atención de salud, que ponen en riesgo la seguridad del paciente.

La insatisfacción laboral es un problema que puede ser atendido desde esta perspectiva, ya que las personas contentas son las que mejores resultados dan.

Reafirmando lo anterior, se puede asegurar que México puede 


\section{Cuadro 2. Componentes Magnéticos del modelo y su alcance ${ }^{1}$}

\section{Componentes}

del Modelo

Liderazgo

de transformación

Empoderamiento

estructural
Fuerzas del Magnetismo

Calidad del liderazgo

de enfermería

Estilo de gestión

\section{Estructura}

de la organización

Programas políticos

y personales

Organización

de la comunidad y sanidad

Imagen de la enfermería

Desarrollo profesional

Modelos de cuidados profesionales

Recursos y consultas

Práctica enfermera profesional ejemplar

Autonomía

\section{Alcance}

Provee el mecanismo para comunicar y automatizar su modelo del proceso de cuidados. Mejora de las gestiones clínicas mediante la visibilidad del cumplimiento, datos y medidas de resultados.

Debe facilitar el manejo de las gestiones clínicas y la efectividad

Toma de decisiones compartida

Foco en el personal operativo

Estructuras de comunicación efectivas

Soporta y permite un modelo de colaboración interdisciplinario de trabajo y comunicación.

Soporta el alcance de la práctica para cada clínico mediante la configuración adecuada.

Permite la presencia de líderes de enfermería en los comités ejecutivos

Aplica el conocimiento sobre la tecnología para el proveedor adecuado en el momento adecuado

Colaboración con otras organizaciones sanitarias

Cruce continuo de información

Historia centrada en la persona

Historia compartida en la comunidad

Historia compartida en la atención domiciliaria

Conciencia interdisciplinar

Mejor comunicación y coordinación en

el equipo de cuidados

La dirección de enfermería es la clave de la influencia organizacional en la toma de decisiones

Soporte a la Decisión y pensamiento crítico Soporte de planes multidisciplinares

Facilita el aprendizaje de Enfermería Basada en Evidencia Capacidad del mapeo de terminologías bajo un único índice

Decisión del estado futuro bajo el uso de la tecnología para maximizar el cuidado individualizado y el elevado nivel de calidad asistencial.

Liderazgo clínico experimentado

Procesos recomendados en las mejores prácticas
Referencias online y links basados en evidencia científica Servicios de conocimiento 


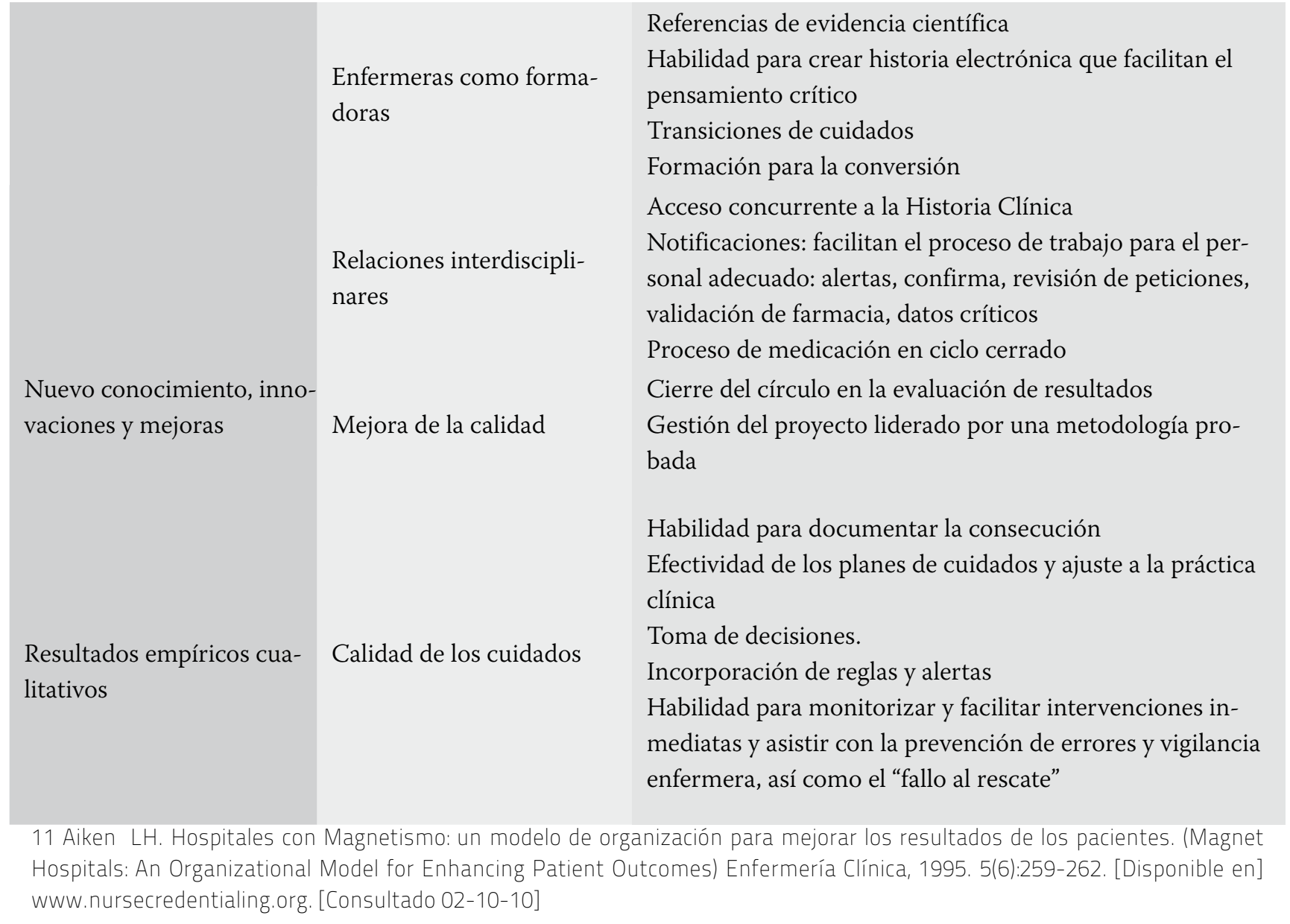

\section{Fig.1 Los 5 \\ Componentes del modelo y las fuerzas del magnetismo.}

Problemas mundiales en el cuidado de la salud

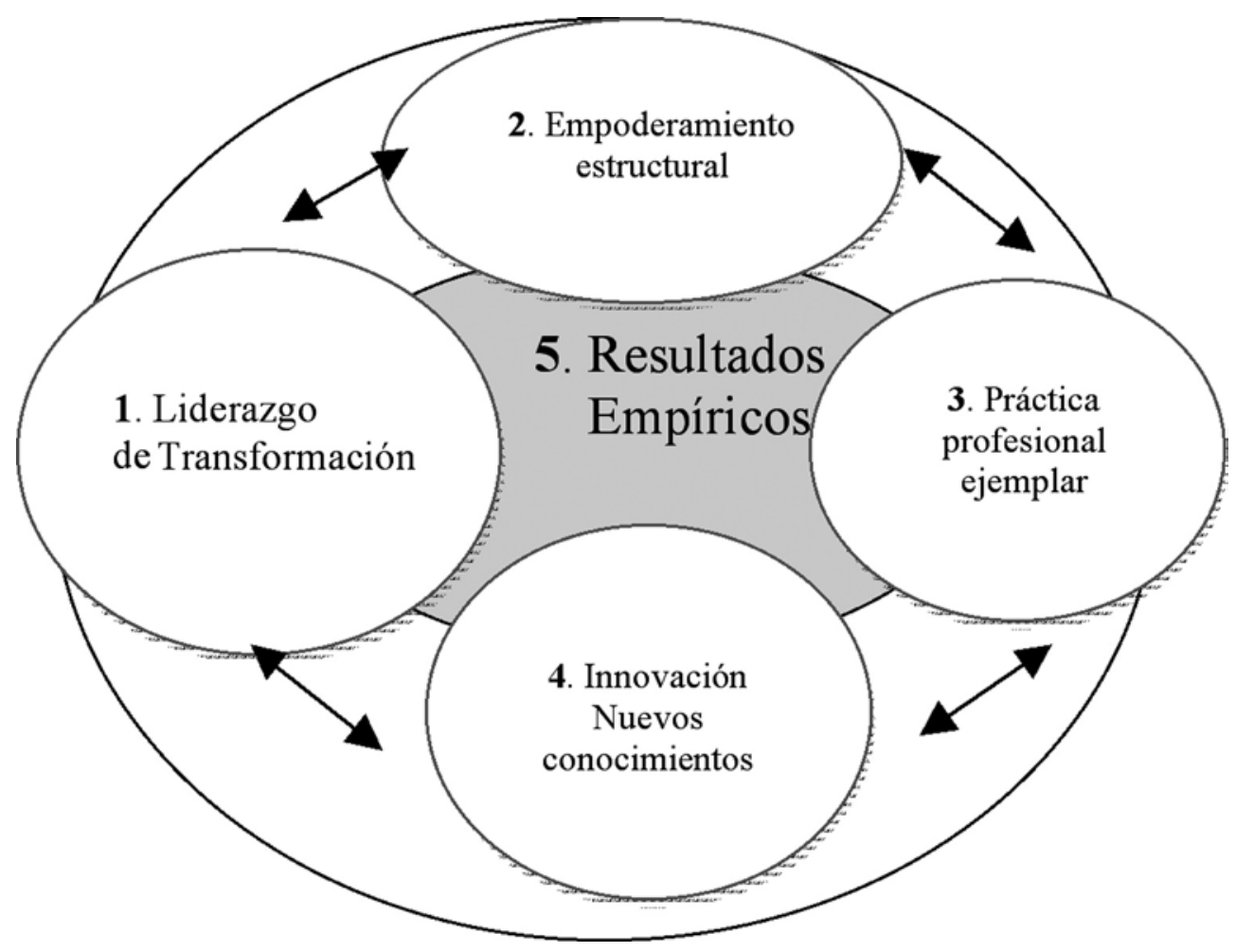


utilizar este modelo para contrarrestar los problemas que se tienen derivados de la práctica, y así poder garantizar cuidados de calidad donde el profesional de enfermería tenga más autonomía, desarrolle un liderazgo que le permita desenvolverse ampliamente ocupando nuevos escenarios y así pueda satisfacer sus propias necesidades laborales. No cabe duda que la propuesta de utilizar este modelo abre nuevos escenarios, sobre todo para el área de investigación.

Esta visión para con las instituciones de salud, fue planteada por Nigthingale ${ }^{13}$ siendo visionaria para su época, al identificar la necesidad del liderazgo para transformar el cuidado. Así lo reiteró en su obra: "Estamos solo en el umbral de la enfermería. En el futuro, que no veré, o que seré anciana, ipuede ser que se abra un camino mejor! Caminos por los cuales cada niño, cada humano, pueda tener la mejor oportunidad para su salud, caminos en que cada persona enferma tenga la mejor oportunidad de recuperarse, métodos que serán aprendidos y practicados."

Con este argumento se puede afirmar que esta visión no está lejos de ser una realidad.

\section{REFERENCIAS BibLIOGRÁFICAS}

1 Bucham J. Magnet hospitals in a changing health care environment. Journal of Advanced Nursing; 1999. 30(1): 100-08.

2 Lake ET. Development of the Practice Environment Scale of the Nursing Work Index. Research in Nursing \& Health; 2002. 25: 176-88.

3 Carrillo de Albornoz G. Análisis comparativo del ausentismo entre la asistencia especializada y la asistencia primaria, para la misma área asistencial. Enfermería Integral;1997. 41: 28-31

4. Aiken LH, et al. Hospital nurses risk of occupational exposure to blood: Prospective, retrospective, and institutional reports. American Journal of Public Health; 1997. 87: 103-07.

5 World Health Organization. Country profiles. 2001. Disponible en: www.who.org. [Consultado el 0510-2010]

6 McClure M, et al. Magnet Hospitals: Attraction Retention of Professional Nurses. Kansas City: American Academy of Nursing; 1983.

7 Cerner.Org. La certificación de Hospital Magnético. Excelencia enfermera, información, tecnología e innovación. Viaje seguro al Hospital Magnético. [Cerner Iberia. 28046] Madrid. Disponible en: www.cerner.es [Consultado 25/03/2010]

8 López Alonso S. Satisfacción de la enfermera y calidad del cuidado. Evidentia 2004 ene-abr: 1(1). Disponible en: http://www.indexf.com/evidentia/n1/6articulo.php [ISSN: 1697-638X]. [Consultado el 05/10/2010]

9 Teresa Faura, et al. Validation of a Measure to Assess the Hospital Clinical Nurse Practice Environment: A Cross National Pilot Study. Enfermería Clínica; 2002 12:13 22 .

10 Avedis Donavedian A. la calidad de la atención médica. La prensa médica mexicana. México 1984.

11 Aiken LH. Hospitales con Magnetismo: un modelo de organización para mejorar los resultados de los pacientes. (Magnet Hospitals: An Organizational Model for Enhancing Patient Outcomes) Enfermería Clínica, 1996. 5(6):259-262. [Disponible en] www.nursecre- dentialing.org. [Consultado 02-1010]

12 García Palacios N. et al. Entorno clínico de la enfermera de hemodiálisis en la provincia de Cádiz. Revista de la Sociedad Española de Enfermería Nefrológica versión impresa ISSN. 2004. Madrid jul.sep. 7 (3); 1139-1375.

13 Dossey B. Holistic Nursing: From Florence Nightingale's Historical Legacy to 21st Century Global Nursing. Alternative Therapies . 2010; 16(5): 14-21 